\title{
Combined Use of Mechanical Thrombectomy with Angioplasty and Stenting for Acute Basilar Occlusions with Underlying Severe Intracranial Vertebrobasilar Stenosis: Preliminary Experience from a Single Chinese Center
}

F. Gao, W.T. Lo, X. Sun, D.P. Mo, N. Ma, and Z.R. Miao

\begin{abstract}
BACKGROUND AND PURPOSE: Acute basilar occlusions have a poor prognosis without recanalization. Many have underlying severe atherosclerotic intracranial stenosis coexisting with acute thrombosis, requiring treatment of both pathologies in the same session, though technical risks may be encountered. The purpose of this study was to evaluate the technical feasibility and safety of combined treatment by using stent retrievers for the thrombosis, together with angioplasty and stent placement for the underlying stenosis.
\end{abstract}

MATERIALS AND METHODS: This was a retrospective review of 13 patients with basilar occlusions treated with thrombectomy by the Solitaire stent retriever and angioplasty and intracranial stent placement for underlying severe vertebrobasilar stenosis in the same session. Reperfusion was assessed in terms of the $\mathrm{TICl}$ score. Perioperative complications were recorded. Clinical outcomes were assessed by the NIHSS at discharge and the mRS on follow-up at 90 days.

RESULTS: Of the 30 patients with acute basilar artery occlusions treated with stent retrievers during the study period, 18 had coexisting severe intracranial stenosis. Thirteen patients meeting the criteria for our study received combined mechanical thrombectomy and angioplasty with stent placement. The successful recanalization rate was 100\%. Distal vessel embolizations occurred in 3 patients. There were 2 mortalities. On discharge, 10 patients (77\%) had an improvement in NIHSS of $\geq 10$ points. At 90 days, 6 patients (46\%) had a good functional outcome with an mRS of $\leq 2$.

CONCLUSIONS: The combined use of mechanical thrombectomy with angioplasty and stent placement for acute basilar occlusions with underlying severe intracranial atherosclerotic stenosis is technically feasible and safe.

ABBREVIATIONS: BAO = basilar artery occlusion; PTA = percutaneous transluminal angioplasty

A cute basilar artery occlusion (BAO) carries a high morbidity and mortality. Among patients treated conventionally with antiplatelets or anticoagulation, the death and dependency rate was $80 \% .{ }^{1}$ Even with intravenous or intra-arterial thrombolysis, the overall death or dependency rate is not much improved. Without recanalization, the mortality can be up to $85 \%-95 \%$ and the likelihood of good outcome was only $2 \%$. $^{2}$ The mechanism of occlusion includes acute local thrombosis over an underlying severe atherosclerotic stenosis and embolization from distant

Received December 15, 2014; accepted after revision March 9, 2015.

From the Department of Interventional Neurology (F.G., X.S., D.P.M., N.M., Z.R.M.), Beijing Tiantan Hospital, Capital Medical University; China National Clinical Research Center for Neurological Diseases; Center of Stroke, Beijing Institute for Brain Disorders; Beijing, China; and Department of Medicine (W.T.L.), Queen Elizabeth Hospital, Hong Kong, China.

Please address correspondence to ZhongRong Miao, MD, Department of Interventional Neurology, Beijing Tiantan Hospital, Capital Medical University, 6 Tiantan Xili, Dongcheng District, Beijing 100050, China; e-mail: zhongrongm@163.com

三 Indicates article with supplemental on-line table.

http://dx.doi.org/10.3174/ajnr.A4364 sources. There has not yet been any epidemiologic data on the proportion of acute BAO attributed to atherosclerotic stenosis in Asians, though deducing from the higher prevalence of intracranial atherosclerosis observed in Asians, and especially in the Chinese population, ${ }^{3}$ the proportion of patients with $\mathrm{BAO}$ and underlying atherosclerotic stenosis may be higher than the 26\%-36\% reported on imaging studies from the white population. ${ }^{4}$ For the treatment of BAO, mechanical thrombectomy with stent retrievers has emerged in recent years as a promising method with a higher recanalization rate and better functional outcome., However, for those with underlying severe stenosis, there have been concerns over the feasibility and safety of these devices: Reperfusion may fail to be achieved by mechanical thrombectomy devices alone in patients with severe stenosis ${ }^{7}$; retrieval of the stent retrievers past the stenosis may damage the endothelium or the atherosclerotic plaque, leading to an increased risk of acute thrombosis and reocclusion; and the resistance posed by the stenosis may cause distortion of the natural course of the vessel during the tug of the retrievers past the stenosis, leading to tearing of 
the perforator vessels and intracerebral hemorrhage. We hereby report our experience with 13 patients treated with a combination of mechanical thrombectomy with the Solitaire device (Covidien, Irvine, California) for the thrombosis and angioplasty with stent placement for the underlying stenosis.

\section{MATERIALS AND METHODS Patients}

This was a retrospective analysis of 30 patients with acute basilar artery occlusion who had undergone endovascular treatment with stent retrievers in our hospital from June 2012 to June 2014. Eighteen of these 30 patients were found to have underlying severe atherosclerotic intracranial vertebrobasilar stenosis. Thirteen patients fulfilling the following criteria were reviewed in this study: 1) an acute BAO presenting within 24 hours of symptom onset; 2 ) an NIHSS score of $\geq 8$ on presentation; 3 ) no established large brain stem or cerebellar infarct on admission imaging studies, defined as involvement of more than two-thirds of the brain stem or cerebellum; 4) underlying severe stenosis of the intracranial vertebrobasilar artery of $\geq 70 \%$ on DSA; 5 ) $\geq 1$ vascular risk factor for atherosclerosis present (eg, diabetes, hypertension); and 6) a premorbid $m R S$ of $\leq 2$. Five patients were excluded in our analysis: 3 due to presentation $>24$ hours from symptom onset with progressive deterioration and 2 due to large brain stem infarct size on admission imaging. The endovascular treatment for the latter 2 patients was performed in the initial phase of our acute stroke interventional service.

All patients had an assessment performed by a stroke neurologist on admission, and the NIHSS score was recorded. Multimodal MR imaging (including DWI, MRA, and MR perfusion studies) was performed on admission unless contraindicated, in which case cerebral CT, CTA, and CTP studies were performed. Baseline demographics, admission NIHSS and mRS scores, symptom-to-door time, needle-to-recanalization time, and door-to-recanalization time were documented. For patients with unknown symptom-onset time, the last known normal-to-onset time was taken as the symptom-to-onset time. The NIHSS score on discharge and the mRS score on follow-up at 90 days were recorded.

This study was approved by the institutional ethics committee, which also waived informed consent due to the retrospective nature of this study.

\section{Interventional Procedure}

With the patient under general anesthesia and by using transfemoral access, we advanced a $6 \mathrm{~F}$ guiding catheter into the V2 segment of the vertebral artery. Intravenous heparin was given, aiming for an activated clotting time of 250-300 seconds. A microcatheter (Rebar microcatheter; Covidien) was carefully navigated through the basilar occlusion over a 0.014-inch microwire (Transend; Stryker, Kalamazoo, Michigan) under fluoroscopic guidance. Angiographic runs via the microcatheter were performed to confirm its location in the target artery with its tip distal to the thrombus. The Solitaire AB device was used for mechanical thrombectomy in our center. After advancement of the Solitaire via the microcatheter to the occluded segment, the stent retriever was unsheathed and allowed full expansion through the throm- bus, acting as a transient endovascular bypass to restore flow across the occluded segment, and angiography was performed to assess the distal vessel flow. The fully deployed Solitaire device was then partially resheathed, and together with the delivery microcatheter, gently pulled back as a single unit, and recovered for thrombectomy. If this failed or recanalization was insufficient, further retrievals would be attempted. Angioplasty and stent placement would be considered if there was underlying severe vertebrobasilar stenosis causing inadequate distal perfusion, or new thrombus formation or reocclusion was noted at the site of residual stenosis on repeat angiography up to 30 minutes after thrombectomy. A loading dose of aspirin, $300 \mathrm{mg}$, and clopidogrel, $300 \mathrm{mg}$, was given via nasogastric tube to patients without prior use of antiplatelets once the decision to proceed to stent placement was made. Pantoprazole was given for gastric protection. Stent placement was done with either the balloon-mounted Apollo stent (MicroPort Medical [Shanghai], Shanghai, China) (Fig 1) or the self-expanding Wingspan stent (manufactured by Stryker for Boston Scientific, Natick, Massachusetts) (Fig 2). These stents have an advantage over detachment of the Solitaire $\mathrm{AB}$ device used as a stent because they provide a stronger radial force to overcome the underlying stenosis. Device selection depends on the vessel characteristics and lesion morphology, as previously described. ${ }^{8}$ For patients with tortuous arterial access and anticipated difficulty for stent passage, only percutaneous transluminal angioplasty (PTA) was performed. If patients were noted to have acute thrombosis over the stent or significant residual thrombosis impeding forward blood flow, glycoprotein IIb/ IIIa inhibitor infusion (tirofiban) would be given.

Follow-up CT was performed immediately postoperatively to exclude intracerebral hemorrhage. Transcranial Doppler, CTA, MRA, or DSA would be performed within 24 hours postintervention to assess the patency of the basilar artery. Patients were maintained on dual-antiplatelet therapy of aspirin, $100 \mathrm{mg}$, and clopidogrel, $75 \mathrm{mg}$, for 3 months, followed by single-antiplatelet therapy life-long. Thromboelastography was arranged to check for platelet function, and CYP219 testing, to check for genetic resistance to clopidogrel. However, initial choice of antiplatelets was not based on these results because the interventions had been performed as an emergency operation and the results were not available before the operation. All patients were put on statins aiming for a low-density lipoprotein of $<1.8$ and on antihypertensives aiming for a systolic blood pressure of $<140 \mathrm{~mm} \mathrm{Hg}$. Patients were followed up at 90 days by clinic visit or by phone if they could not attend follow-up. Follow-up imaging by CT angiography was arranged.

\section{Outcome Measures}

The primary outcome measure was the successful reperfusion rate, defined as a TICI grade $2 \mathrm{~b}$ or 3 after endovascular treatment. The other outcome measures recorded were the intraoperative complications, including vessel perforation, arterial dissection, symptomatic intracerebral hemorrhage, and any other in-hospital neurologic complications. Clinical outcomes were the NIHSS score on discharge, functional outcome, and vascular-related mortality at 90 days. A good functional outcome was defined as an $\mathrm{mRS}$ of $\leq 2$. 

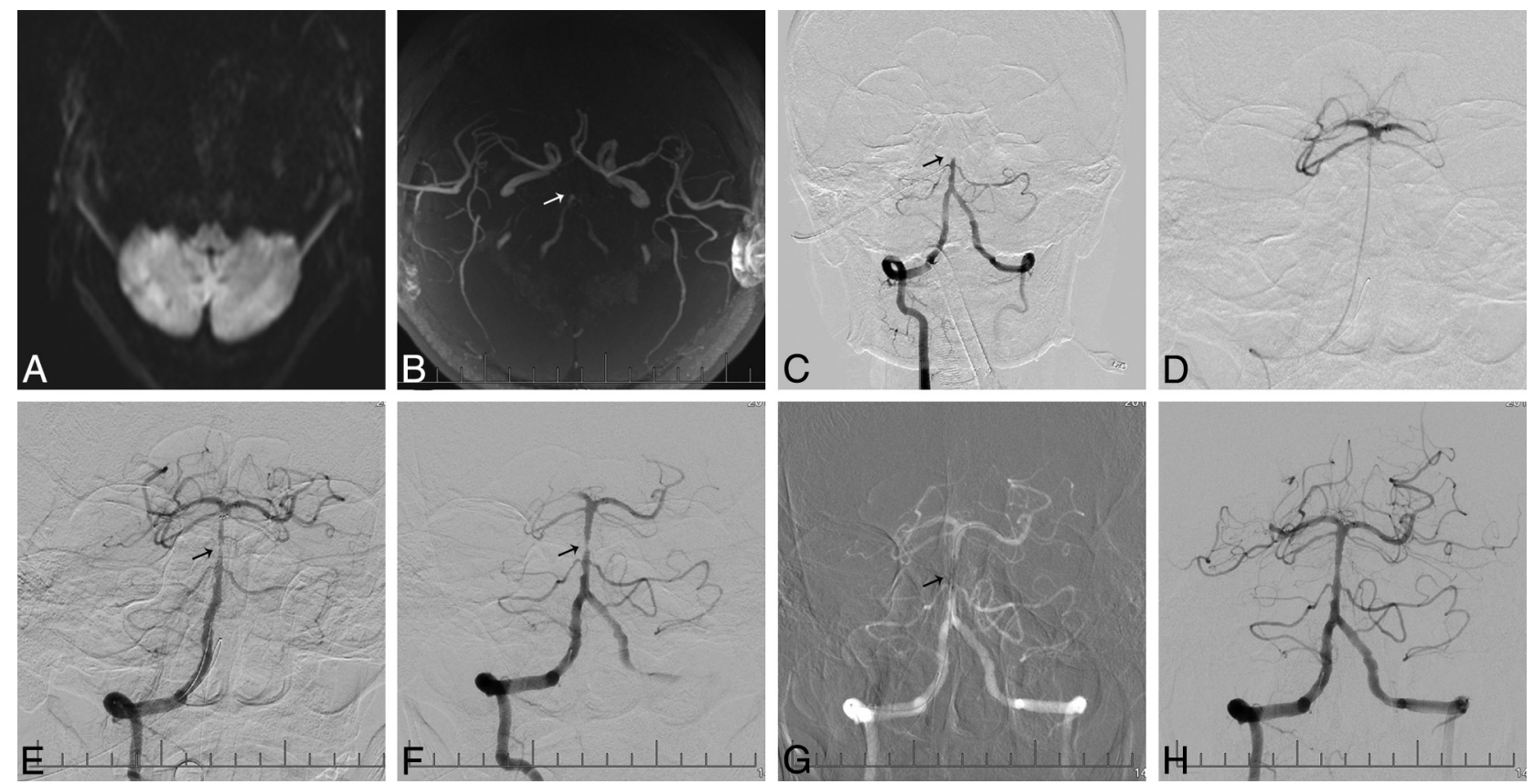

FIG 1. A 54-year-old male patient who presented with vertigo and decreased consciousness for 10 hours. $A$, MR imaging shows multiple infarcts over the bilateral cerebellar hemispheres on DWI. B, MRA shows basilar artery occlusion (arrow). C, A right vertebral angiogram shows occlusion of the basilar artery (arrow).D, An angiogram after crossing of the basilar artery occlusion with a microcatheter shows patent distal flow at the basilar artery tip, with opacification of both posterior cerebral arteries and superior cerebellar arteries. E, Angiogram after deployment of the Solitaire AB device shows restoration of flow in the basilar artery, with suspected thrombus and focal stenosis (arrow) in the mid-distal segment of the basilar artery. $F$, Angiogram post-mechanical thrombectomy shows underlying severe focal stenosis of the basilar artery (arrow). $G$, Deployment of an Apollo stent (arrow) at the site of the basilar artery stenosis. $\mathrm{H}$, Final angiography shows a $\mathrm{TICl}$ flow of grade 3 in the basilar artery with good distal perfusion.
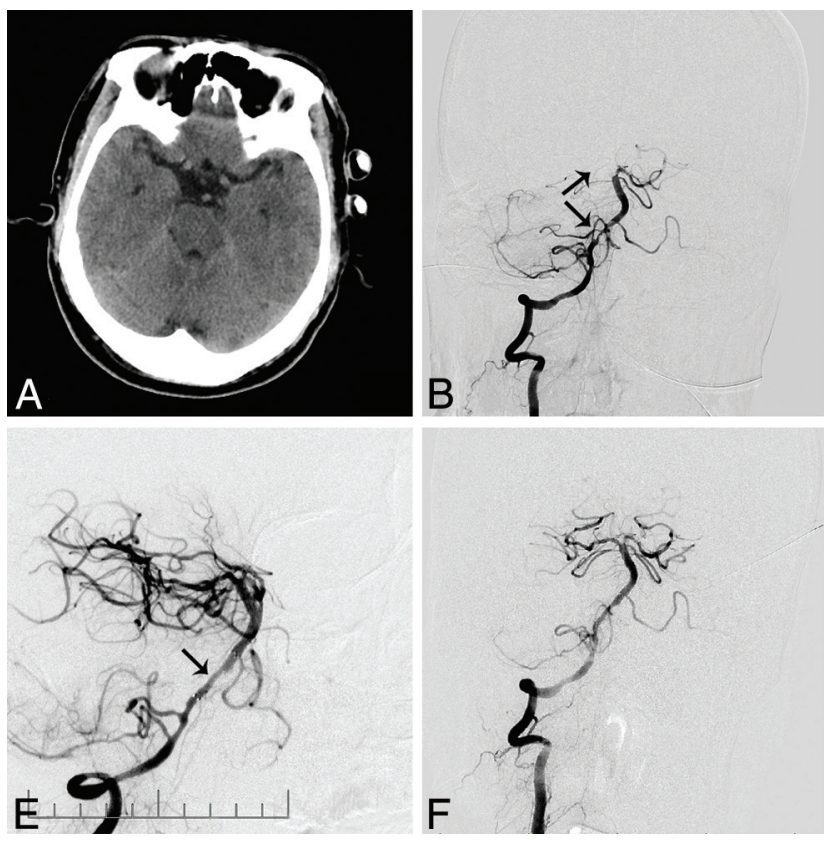
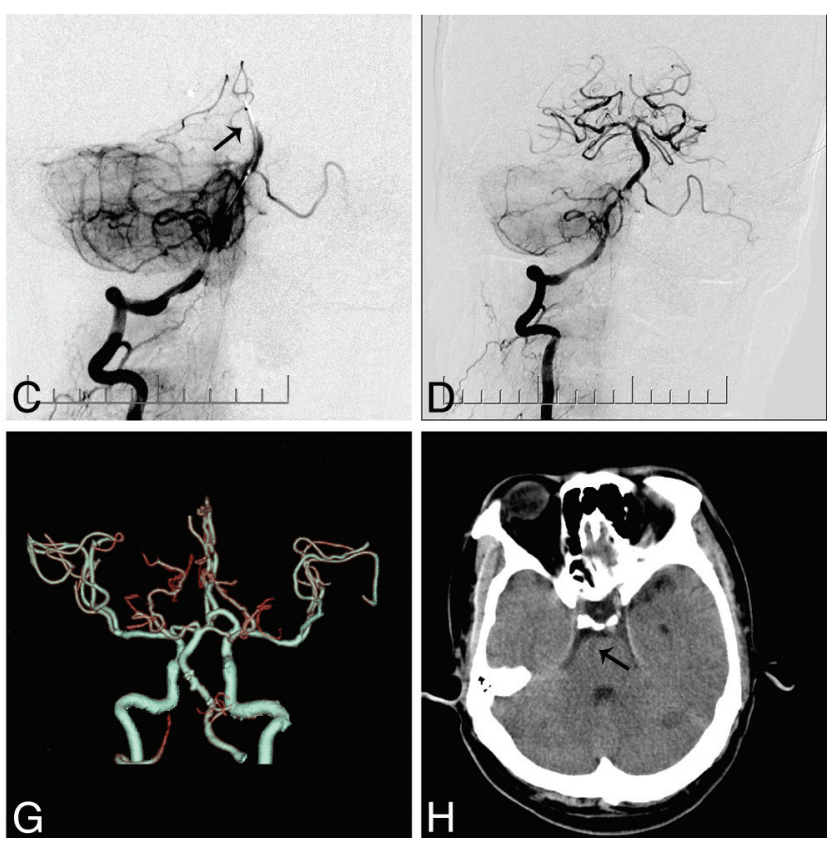

FIG 2. A 55-year-old male patient who presented with tetraparesis and decreased consciousness for 6 hours. A, Plain CT on admission does not reveal any large territory infarct. $B$, The right vertebral angiography shows occlusion at the tip of the basilar artery (arrow) and right posterior cerebral artery and severe stenosis of the right vertebrobasilar junction (arrows). $C$, Angiography postdeployment of the Solitaire AB device at the proximal right posterior cerebral artery shows no opacification of the distal basilar artery (arrow). $D$, Angiography post-mechanical thrombectomy shows opacification of the distal basilar artery and both posterior cerebral arteries and severe stenosis in the vertebrobasilar junction. $E$, Deployment of a Wingspan stent (arrow) at the vertebrobasilar junction. $F$, Right vertebral angiography after stent placement shows resolution of the proximal basilar artery stenosis and good contrast flow in the basilar artery (TICl grade 3). G-H, Follow-up CT and CTA at 24 hours show a patent basilar artery and a small right pontine infarct. 
Summary of patient characteristics, treatment, and outcome

No. of Patients

$(N=13)$

Male sex

Age (mean $\pm \mathrm{SD})(\mathrm{yr})$

Pretreatment NIHSS (mean \pm SD)

Symptom-to-door time (mean $\pm \mathrm{SD}$ ) (min)

Needle-to-recanalization (mean $\pm \mathrm{SD})(\mathrm{min})$

Door-to-reperfusion (mean $\pm \mathrm{SD})(\mathrm{min})$

Underlying site of stenosis

BA

V4

Endovascular treatment for the stenosis

PTA alone

Apollo stent

Wingspan stent

Glycoprotein Ilb/IIla inhibitor

Pre-PTA/stenting stenosis (mean $\pm \mathrm{SD}$ )

Post-PTA/stenting stenosis (mean $\pm \mathrm{SD}$ )

Pre-PTA/stenting TICl (post-mechanical thrombectomy)

0

1

$2 \mathrm{a}$

Post-PTA/stenting TICl

$2 b$

3

Distal embolization to PCA/cerebellar arteries

Outcome

NIHSS score on discharge for surviving patients (mean $\pm \mathrm{SD})$

$\%$ of patients with NIHSS $\geq 10$-point improvement

Mortality on discharge

$\mathrm{mRS}$ at 90 days

$\mathrm{mRS} 0-2$

mRS 0-3

Ischemic events at 90-day follow-up

Note:-BA indicates basilar artery; $\mathrm{PCA}$, posterior cerebral artery; $\mathrm{V} 4$, vertebral artery V4 segment.

\section{RESULTS}

Eighteen of the 30 patients $(60 \%)$ with acute BAO in our study who were treated with stent retrievers had underlying severe intracranial vertebrobasilar stenosis. Thirteen patients satisfied our study criteria. The mean age was 56 years. The mean baseline NIHSS score was 26 (range, 19-34). All patients presented outside the time window for intravenous thrombolysis, apart from 1 inpatient with known basilar stenosis deteriorating acutely during admission; hence, endovascular therapy was arranged immediately. The mean symptom onset-to-door, needle-to-recanalization, and door-to-recanalization times were 587, 93, and $301 \mathrm{~min}-$ utes, respectively (Table and On-line Table).

After initial mechanical thrombectomy (average, 1.4 passes), all 13 patients had a TICI score of $\leq 2 \mathrm{a}$. Because satisfactory reperfusion was not achieved and underlying severe stenosis was noted, 10 patients had stent placement performed and 3 received PTA alone due to tortuous artery access. The site of the underlying severe stenosis was the basilar artery for 10 patients and the vertebral artery V4 segment for 3 patients. Six patients were given supplementary glycoprotein IIb/IIIa inhibitor for acute new thrombosis or significant residual thrombosis noted. The overall rate of successful reperfusion (final TICI 2 b to 3 ) was $100 \%$. The mean residual stenosis was $24.6 \%$. Three patients had distal em- bolization to the posterior cerebral arteries or the cerebellar arteries on angiography during the procedure. No patient had vessel rupture or dissection. There were 2 mortalities. One patient developed a large-territory infarct and cerebral edema. Another patient died due to severe gastrointestinal bleeding possibly related to reactive gastritis aggravated by the use of dual antiplatelets. On discharge, an improvement of the NIHSS score of $\geq 10$ points was noted for 10 patients (76.9\%). No patients were lost to follow-up. At 90 days, 6 patients had a good functional outcome with an mRS $\leq 2$, which represented $46 \%(6 / 13)$ of all patients and $54.5 \%$ (6/ 11 ) of the survivors. None of the patients developed recurrent ischemic attacks. Five patients had follow-up imaging by CT angiography at 90 days, with no restenosis or reocclusion demonstrated. All patients had CYP2C19 checked for clopidogrel resistance, and all had normal findings. Four patients had thromboelastography testing, and none showed aspirin resistance.

For the 5 patients who did not meet the inclusion criteria for our study, all had successful recanalization after thrombectomy and stent placement. One patient with a large brain stem infarct on admission imaging further deteriorated and died on day 2. Follow-up imaging of this patient showed cerebral edema with no hemorrhagic transformation. For the 4 patients who survived, 1 patient had an mRS of 2 and 3 patients had an $m R S$ of $>3$ on follow-up at 90 days.

\section{DISCUSSION}

This case series selectively studied 13 patients with acute basilar occlusions, requiring combined mechanical thrombectomy for the thrombosis and stent placement for the underlying stenosis, performed in the same session. The prognosis of acute BAO in general is poor. Current studies have reported an improved outcome achieving a recanalization rate from $74 \%$ to $100 \%$ in recent years with the use of stent retrievers, ${ }^{9,10}$ but these studies were on acute BAO in general and the outcome of the patients had not been stratified according to whether there was underlying severe stenosis, probably due to the small absolute number of patients in the studies. ${ }^{9,11-14}$ The exact incidence of acute BAO with underlying severe stenosis is unknown. Extrapolation from case reports on patients with acute BAO treated by endovascular means requiring additional angioplasty or stent placement for underlying severe intracranial stenosis showed an approximate incidence of $14 \%-29 \%$ in studies of the white population..$^{9,11-13}$ In our registry, having underlying stenosis was not uncommon; 60\% (18/30) had severe intracranial stenosis requiring additional angioplasty or stent placement. We believe that other centers will also encounter similar patients, especially when treating patients from Asian countries where intracranial stenosis is more prevalent. ${ }^{3}$

Patients with combined thrombosis and underlying stenosis represent a distinct group in management, because they have a different technical risk profile and a good outcome is dependent on the treatment of both the thrombosis and the severe stenosis. All 13 patients in our analysis had the stent placement performed in the same session as the thrombectomy, rather than as a staged operation, because they could not achieve timely satisfactory reperfusion after thrombectomy alone without treatment of the stenosis. Possible reasons for failure to attain good reperfusion included flow limitation by the proximal stenosis or new thrombus 
formation possibly aggravated by endothelial injury from the passage of the mechanical thrombectomy device through the stenosis. The negative Stenting vs Aggressive Medical Management for Preventing Recurrent Stroke in Intracranial Stenosis trial, which had raised concerns about the safety of intracranial stent placement, ${ }^{15}$ had been a study on secondary stroke prevention for a group of relatively more stable patients for whom a low operative risk was imperative to gain benefit over medical treatment. However, for our patients with BAO presenting acutely with severe neurologic deficits and a high risk of further deterioration and death if timely reperfusion could not be achieved, angioplasty and stent placement could be regarded as a brain- or life-saving attempt for this group with poor prognosis. Moreover, the potential immediate endothelial damage after the passage of the mechanical thrombectomy device past the stenosis might lead to an increased risk of early reocclusion if stent placement was not attempted or stalled.

For our patients, thrombectomy was first performed with stent retrievers, followed by angioplasty or stent placement for the residual severe stenosis. In contrast to those patients with purely thromboembolic occlusions, there were theoretic concerns that the retrieval of the thrombectomy devices may be impeded by the stenosis and forceful maneuvers to retrieve these devices would increase the risk of vessel rupture or tearing of perforator vessels. ${ }^{16}$ We did not encounter such difficulties in our study patients. Similarly, no retrieval difficulties or device complications were reported in the 4 patients with underlying severe stenosis in the study of Mordasini et al, ${ }^{12}$ of whom 3 had stent placement and 1 patient had only PTA performed, and in the 5 patients with stent placement in the study of Espinosa de Rueda et al $^{11}$; but it is likely that publication bias existed.

In choosing stents for the underlying severe intracranial stenosis, 11 patients received the Wingspan stent after prior angioplasty or the Apollo stent according to lesion and vessel characteristics, which provided a relatively stronger radial force than a detached Solitaire AB stent. ${ }^{17}$ Deployment of these stents served several purposes: First, stent placement could improve distal perfusion, decreasing the risk of thrombosis induced by stagnant flow and facilitating the spontaneous thrombolysis of distal emboli. Second, the shear from the passage of the retrievers past the stenosis could potentially damage the endothelium or the unstable atherosclerotic placque, increasing the risk of reocclusion related to the increased platelet activation and aggregation and vessel dissection. Stent placement over these vulnerable structures in the same session aimed to decrease the risk of thrombosis, recoil, restenosis, and dissection. Stent placement has advantages over angioplasty alone in reducing such risks because it can provide better coverage of the lesion and exposed endothelium. However, for some patients with tortuous access leading to a higher risk of complications if stent placement was attempted, angioplasty alone was performed.

In our study, the technical success rate for reperfusion was $100 \%$. There was satisfactory resolution of the stenosis without recoil or dissection in all patients. In the case series of Möhlenbruch et al, ${ }^{9}$ in 24 patients treated with mechanical thrombectomy, the rate of successful reperfusion was $75 \%$ when only thrombectomy was performed; after stent placement was per- formed for the 7 patients with underlying intracranial stenosis, the overall reperfusion rate improved to $87.5 \%$. Other studies have reported technical success rates in the range of $74 \%-$ $94 \% .^{11,12,14,18}$ No patients had procedure-related intracerebral hemorrhage on postoperative imaging; these results compared favorably with the $14 \%$ intracerebral hemorrhage rate in the endovascular treatment group from a recent meta-analysis. ${ }^{19}$ There were 2 mortalities, neither of which were procedure-related. On discharge, an improvement of NIHSS score of $\geq 10$ points was noted for 10 patients $(76.9 \%)$. This compared favorably with the $35.7 \%$ of patients having an NIHSS improvement at 24 hours in the study of Mordasini et al, ${ }^{12}$ and the $54 \%$ with improvement on discharge in the study of Möhlenbruch et $\mathrm{al}^{9}$ on patients with $\mathrm{BAO}$ in general. At 90 -day follow-up, a good functional outcome of $\mathrm{mRS} \leq 2$ was achieved in 6 of 13 patients (46.1\%). Möhlenbruch et al reported an overall favorable clinical outcome of $33 \%$, while Andersson et $\mathrm{al}^{13}$ reported an overall 57\% favorable outcome for their 28 patients.

Other smaller case series reported a rate of $29 \%-50 \%$ on BAO treatment by using stent retrievers. ${ }^{11,12,14,18}$ However, it is difficult to do a reliable comparison of our results with those in other acute $\mathrm{BAO}$ series with mechanical thrombectomy performed due to the heterogeneity in patient characteristics, inclusion criteria, and types of adjuvant therapy used. The patients in our study were relatively young, with a mean age of 56 years. Younger age is a favorable prognostic factor because these patients have less challenging anatomy and are more likely to have better outcomes than older patients. However, our patients had a high NIHSS score on admission and a long symptom-to-recanalization time; this might account for the limited functional outcome in some patients. Compared with patients with good functional outcome, patients with poorer outcome of $\mathrm{mRS}$ of $>2$ had a higher NIHSS score and longer symptom-to-door, needle-to-recanalization, and door-torecanalization times. Reasons for the relatively long door-to-recanalization time included the acute interventional stroke service workflow in our institution being in the process of optimization during the initial stages and delay in receiving general anesthesia and intubation required by our patients for airway protection and aspiration prevention. Compared with patients with no underlying severe stenosis for whom sole mechanical thrombectomy was already successful in obtaining adequate distal perfusion, our patients with severe stenosis required additional time for the steps of angioplasty and stent placement to achieve reperfusion.

There were limitations to this study. This was a retrospective study with a small sample size of 13 patients providing preliminary experience of combined treatment, but further large-scale prospective studies with a longer follow-up are warranted. Because our patients could not achieve satisfactory reperfusion of the distal territories after the first stage of mechanical thrombectomy, revascularization by angioplasty or stent placement of the underlying stenosis in the same session was performed.

There was no control group of patients with just conservative treatment given for the stenosis, and there are currently sparse reports in the literature specifically on the outcome of patients with underlying severe stenosis for comparison. Only with randomized trials would we be able to directly compare the outcome of patients with acute $\mathrm{BAO}$ with or without stent placement per- 
formed in the same session for the underlying stenosis. We recognized that intracranial stent placement with the use of the Wingspan stent would be regarded as off-label in some countries after the publication of the Stenting vs Aggressive Medical Management for Preventing Recurrent Stroke in Intracranial Stenosis results. ${ }^{15}$ However, there have been controversies over the study; with improved patient and device selection and increased technical experience, intracranial stent placement still holds a promising role for selected patients. ${ }^{8,20,21}$ The use of the Wingspan stent was not off-label in our center. Our choice of endovascular devices was based on lesion or vessel characteristics ${ }^{8}$; hence, we advise caution against overinterpretation of our data because our study was not meant to compare the efficacy among different vascularization methods by using the Apollo, the Wingspan stent, or sole angioplasty.

Conclusions drawn about which method conveyed a higher success rate would be difficult because the baseline vessel and lesion characteristics were different among patients, and our study was not adequately powered to perform a meaningful statistical interpretation. A previous study reported on the revascularization results of these 2 stents and angioplasty, ${ }^{8}$ though larger trials would be warranted. The use of glycoprotein IIb/IIIa inhibitor was not yet standardized in our hospital or according to international guidelines; more study results are awaited to guide its use. The Solitaire FR stent (Covidien) was not available in our center during the time of the study; instead the Solitaire AB device was used. Balloon occlusion catheters were not available in our center, but until now, there has been no conclusive evidence to prove that the balloon occlusion catheter can be of use in the posterior circulation to decrease the distal embolization rate significantly. The door-to-recanalization time for the patients in our study was relatively long. With more experience in acute stroke intervention gained from the previous years, our institution has streamlined our triage system and workflow to decrease our doorto-recanalization time.

\section{CONCLUSIONS}

Combined mechanical thrombectomy with stent retrievers and angioplasty and stent placement for the treatment of acute basilar occlusion with underlying severe intracranial vertebrobasilar stenosis was safe and feasible. No increased rate of arterial rupture or reocclusion was noted from this study. Further larger prospective clinical trials are needed to confirm our study results.

\section{REFERENCES}

1. Schonewille WJ, Algra A, Serena J, et al. Outcome in patients with basilar artery occlusion treated conventionally. J Neurol Neurosurg Psychiatry 2005;76:1238-41

2. Lindsberg PJ, Mattle HP. Therapy of basilar artery occlusion: a systematic analysis comparing intra-arterial and intravenous thrombolysis. Stroke 2006;37:922-28

3. Wong LK. Global burden of intracranial atherosclerosis. Int J Stroke 2006;1:158-59
4. Mattle HP, Arnold M, Lindsberg PJ, et al. Basilar artery occlusion. Lancet Neurol 2011;10:1002-14

5. Saver JL, Jahan R, Levy EI, et al; SWIFT Trialists. Solitaire flow restoration device versus the Merci retriever in patients with acute ischaemic stroke (SWIFT): a randomised, parallel-group, non-inferiority trial. Lancet 2012;380:1241-49

6. Nogueira RG, Lutsep HL, Gupta R, et al; TREVO 2 Trialists. Trevo versus Merci retrievers for thrombectomy revascularisation of large vessel occlusions in acute ischaemic stroke (TREVO 2): a randomised trial. Lancet 2012;380:1231-40

7. Park JH, Park SK, Jang KS, et al. Critical use of balloon angioplasty after recanalization failure with retrievable stent in acute cerebral artery occlusion. J Korean Neurosurg Soc 2013;53:77-82

8. Miao Z, Song L, Liebeskind DS, et al. Outcomes of tailored angioplasty and/or stenting for symptomatic intracranial atherosclerosis: a prospective cohort study after SAMMPRIS. J Neurointerv Surg 2015;7:331-35

9. Möhlenbruch M, Stampfl S, Behrens L, et al. Mechanical thrombectomy with stent retrievers in acute basilar artery occlusion. AJNR Am J Neuroradiol 2014;35:959-64

10. Mortimer AM, Bradley M, Renowden SA. Endovascular therapy for acute basilar artery occlusion: a review of the literature. J Neurointerv Surg 2012;4:266-73

11. Espinosa de Rueda M, Parrilla G, Zamarro J, et al. Treatment of acute vertebrobasilar occlusion using thrombectomy with stent retrievers: initial experience with 18 patients. AJNR Am J Neuroradiol 2013;34:1044-48

12. Mordasini P, Brekenfeld C, Byrne JV, et al. Technical feasibility and application of mechanical thrombectomy with the Solitaire FR revascularization device in acute basilar artery occlusion. AJNR Am J Neuroradiol 2013;34:159-63

13. Andersson T, Kuntze Söderqvist $\AA$, Söderman M, et al. Mechanical thrombectomy as the primary treatment for acute basilar artery occlusion: experience from 5 years of practice. J Neurointerv Surg 2013;5:221-25

14. Mourand I, Machi P, Milhaud D, et al. Mechanical thrombectomy with the Solitaire device in acute basilar artery occlusion. J Neurointerv Surg 2014;6:200-04

15. Chimowitz MI, Lynn MJ, Derdeyn CP, et al; SAMMPRIS Trial Investigators. Stenting versus aggressive medical therapy for intracranial arterial stenosis. N Engl J Med 2011;365:993-1003

16. Kim TK, Rhim JK, Lee CJ, et al. The limitations of thrombectomy with Solitaire $A B$ as first-line treatment in acute ischemic stroke: a single center experience. J Cerebrovasc Endovasc Neurosurg 2012; 14:203-09

17. Krischek O, Miloslavski E, Fischer S, et al. A comparison of functional and physical properties of self-expanding intracranial stents [Neuroform3, Wingspan, Solitaire, Leo+, Enterprise]. Minim Invasive Neurosurg 2011;54:21-28

18. Roth C, Mielke A, Siekmann R, Ferbert A. First experiences with a new device for mechanical thrombectomy in acute basilar artery occlusion. Cerebrovasc Dis 2011;32:28-34

19. Kumar G, Shahripour RB, Alexandrov AV. Recanalization of acute basilar artery occlusion improves outcomes: a meta-analysis. J Neurointerv Surg 2014 Sep 30. [Epub ahead of print]

20. Miao Z. Intracranial angioplasty and stenting before and after SAMMPRIS: "from simple to complex strategy-the Chinese experience." Front Neurol 2014;5:129

21. Yu SC, Leung TW, Lee KT, et al. Angioplasty and stenting of intracranial atherosclerosis with the Wingspan system: 1-year clinical and radiological outcome in a single Asian center. J Neurointerv Surg 2014;6:96-102 